\title{
Estudo comparativo do desempenho visual e análise de frente de onda entre as lentes intra-oculares multifocais difrativas Tecnis ${ }^{\circledR}$ ZM900 e AcrySof ${ }^{\circledR}$ ReSTOR $^{\circledast}$ SN60D3
}

\author{
Comparison between OPD-Scan results and visual outcomes of Tecnis ${ }^{\otimes}$ ZM900 \\ and ReSTOR ${ }^{\circledast}$ SN6OD3 diffractive multifocal intraocular lenses
}

\author{
Wilson Takashi Hida ${ }^{1}$ \\ Antonio Francisco Pimenta Motta ${ }^{2}$ \\ Newton Kara-José Júnior ${ }^{3}$ \\ HumbertoCosta ${ }^{4}$ \\ Clayton Tokunag'a ${ }^{5}$ \\ Livio Neiva Cordeiro ${ }^{6}$ \\ Daniela Gemperliz \\ Celso Takashi Nakanos
}

\begin{tabular}{|l|}
\hline RESUMO \\
\hline Objetivos: Comparar o desempenho visual e a análise de frente de onda \\
entre as lentes intra-oculares (LIOs) multifocal difrativa Tecnis ${ }^{\circledR}$ ZM900 \\
asférica e AcrySof ${ }^{\circledR}$ ReSTOR ${ }^{\circledR}$ esférica SN60D3. Métodos: Trata-se de \\
estudo prospectivo comparativo que incluiu 78 olhos de 39 pacientes. A \\
avaliação oftalmológica contou com medida da acuidade visual para \\
longe, intermediária e curta distância, sem correção e com a melhor \\
correção óptica, teste de sensibilidade ao contraste e análise de frente de \\
onda por meio do aberrômetro OPD-Scan. Resultados: Acuidade visual \\
para longe e para perto sem correção óptica e análise de aberrometria \\
foram estatisticamente semelhantes em relação a ambas as LIOs; jáa visão \\
intermediária e a sensibilidade ao contraste em condições fotópicas \\
revelaram diferença estatisticamente significante entre as duas lentes a \\
favor da Tecnis ZM900. Conclusão: Ambas as lentes intra-oculares \\
promoveram excelente visão para longe e para perto em situações de alto \\
contraste. A LIO Tecnis multifocal necessita de menos luminosidade para \\
visão em situações de alto contraste, apresenta menos aberrações ópticas \\
e melhor visão intermediária que a LIO ReSTOR.
\end{tabular}

Descritores: Lentes intra-oculares; Sensibilidades de contraste; Visão; Acuidade visual

\section{INTRODUÇ̃̃O}

Um dos principais desafios da moderna cirurgia de catarata é a recuperação da capacidade visual para perto. Muitas formas de abordagem com o objetivo de correção da presbiopia foram tentadas, como monovisão e miopização contralateral ${ }^{(1-2)}$.

As lentes intra-oculares (LIOs) multifocais tentam compensar a eventual perda de estereopsia e visão binocular que limitavam as estratégias usadas com as LIOs monofocais. Apesar de se mostrarem efetivas na visão de perto e de longe, uma limitação destas lentes, sejam refrativas ou difrativas, é a dispersão de energia, que leva à formação de halos e "glare" e à redução da sensibilidade ao contraste ${ }^{(1-7)}$.

As modificações no cristalino que ocorrem com o passar do tempo levam à diminuição da sensibilidade ao contraste ${ }^{(8)}$. A qualidade da visão é ainda mais prejudicada quando se desenvolve catarata. Ao se submeterem à facectomia, os pacientes têm expectativas de melhora da sua visão, com conseqüente 
melhora na qualidade de vida ${ }^{(9)}$. Pensando em satisfazer essas expectativas com uma boa visão funcional, várias inovações nas lentes intra-oculares foram desenvolvidas e os cirurgiões de catarata dispõem de inúmeras opções para escolher ${ }^{(10)}$.

Com a facoemulsificação e o implante de LIO realizados por meio de incisões cada vez menores, possibilitou-se rápida recuperação visual com índices baixos de complicações nas mãos de cirurgiões experientes, além de boa qualidade da visão no período pós-operatório. A avaliação da qualidade da visão pode ser feita por meio de testes variados, como aqueles que avaliam a sensibilidade ao contraste (SC), que consiste na habilidade de distinguir os detalhes das imagens, fato que é dependente da luminosidade do ambiente ${ }^{(11-15)}$.

Buscando entender ainda mais o sistema óptico, novas tecnologias foram desenvolvidas, como a análise da frente de onda, usando a aberrometria de Hartmann-Shack e o "Optical Path Difference", que medem a distância que um feixe luminoso percorre atravessando as diferentes estruturas do olho, conseguindo, assim, medir as aberrações do sistema óptico ${ }^{(16-17)}$

Com o melhor entendimento das aberrações visuais e o intuito de melhorar ainda mais a qualidade da visão do paciente pseudofácico, modificações nas LIOs foram realizadas. Corrigindo as aberrações de alta ordem, por meio da redução na espessura central das LIOs, e com a implementação de superfície asférica foi possível alcançar melhor qualidade na visão após a cirurgia de catarata ${ }^{(15,18-21)}$.

O objetivo deste estudo foi comparar o desempenho visual e a análise de frente de onda das lentes intra-oculares multifocais difrativas Tecnis MF Z900 asférica e a AcrySof ReSTOR SN60D3 esférica.

\section{MÉTODOS}

Este estudo foi conduzido de acordo com as normas éticas para pesquisa clínica e cirúrgica e foi aprovado pela Comissão de Ética para Análise de Projetos de Pesquisa (CAPPesq) da Diretoria Clínica do Hospital das Clínicas e da Faculdade de Medicina da Universidade de São Paulo - Aprovação número 656/06.

Foi um estudo prospectivo, comparativo e não randomizado, em que foram avaliados 78 olhos de 39 pacientes selecionados entre março de 2006 e setembro de 2007. Os critérios de inclusão foram: idade entre 45 e 65 anos; presença de catarata senil bilateral; astigmatismo corneano menor que 1,00 dioptria em ambos os olhos; diâmetro pupilar de no mínimo 3,5 mm em condições mesópicas, medido pelo pupilômetro de Colvard (Oasis Corporation, Glendora, CA, USA); ausência de quaisquer outras doenças oculares, cirurgia ocular prévia, uso de medicações hipotensoras tópicas ou outras doenças sistêmicas que poderiam afetar o desempenho da visão pós-operatória, com diminuição da sensibilidade ao contraste, como a retinopatia causada pelo diabetes mellitus. Os pacientes que preenchiam os critérios de inclusão foram informados sobre a necessidade de exames complementares para medida das aberrações ópticas e sensibilidade ao contraste e somente foram incluídos aqueles que concordaram com a realização deles. Critérios de exclusão foram: complicações intra ou pós-operatórias, dúvidas quanto ao implante da LIO dentro do saco capsular ou descentração da LIO maior que $0,5 \mathrm{~mm}$ avaliada pelo exame à lâmpada de fenda.

A avaliação oftalmológica foi realizada em todas as visitas antes e depois da cirurgia e incluiu: acuidade visual com e sem correção para perto $(33 \mathrm{~cm})$, intermediária $(70 \mathrm{~cm})$ e para longe (6 m) com a tabela de ETDRS (Early Treatment Diabetic Retinopathy Study chart, Precision Vision, Aurora, Colorado, USA), medida da pressão intra-ocular e fundoscopia. As visitas após a cirurgia ocorreram no primeiro, terceiro, sétimo e nonagésimo dias de pós-operatório. Os pacientes foram submetidos à facoemulsificação convencional com implante da lente intra-ocular multifocal asférica Tecnis ${ }^{\circledR}$ MF (ZM900) em 46 olhos de 23 pacientes ou da lente intra-ocular multifocal apodizada esférica Acrysof ${ }^{\circledR} \operatorname{ReSTOR}^{\circledR}$ (SN60D3) em 32 olhos de 16 pacientes.

A LIO multifocal Tecnis ${ }^{\circledR}$ modelo ZM900 (Advanced Medical Optics - AMO, Santa Ana, CA, EUA) é baseada na incorporação da plataforma anterior asférica prolada e posterior difrativa, constituída de polimetilsiloxane. Apresenta $13 \mathrm{~mm}$ de diâmetro total e $6 \mathrm{~mm}$ de óptica biconvexa, com zonas difrativas para visão de perto e longe. Essa LIO apresenta 20 zonas difrativas, com poder de adição de $+4,00$ dioptrias esféricas (DE) na refração plana da LIO, o que corresponde a +3,20 DE na refração plana dos óculos.

A LIO multifocal AcrySof ${ }^{\circledR}$ ReSTOR $^{\circledR}$ modelo SN60D3 (Alcon Laboratories, Fort Worth, TX, EUA) é uma combinação de copolímero de metacrilato acrílico com proteção cromófora química ultravioleta. Apresenta $13 \mathrm{~mm}$ de diâmetro total e $6 \mathrm{~mm}$ de óptica biconvexa, com zonas refrativas para visão de longe e difrativas para visão de perto e longe. Essa LIO apresenta 12 zonas difrativas apodizadas nos 3,6 mm centrais, com poder de adição de +4,00 DE na refração plana da LIO, o que corresponde a +3,20 DE na refração plana dos óculos ${ }^{(19,22)}$.

Todos os pacientes selecionados tiveram poder dióptrico da LIO calculado mediante técnica ultra-sônica de imersão ${ }^{(23)}$, realizada por um único examinador experiente (A.F.P.M.), usando o biômetro ultra-sônico de imersão Ocuscan RXP (Alcon Laboratories, Fort Worth, TX, USA) e o biômetro óptico IOLMaster (Carl Zeiss Meditec, Jena, Germany). As fórmulas usadas para selecionar o poder da LIO foram Hoffer-Q (comprimento axial menor que $22 \mathrm{~mm}$ ) e SRK/T (comprimento axial maior que $22 \mathrm{~mm}$ ), com refração alvo pós-operatória entre 0,1 e 0,4 DE.

Todas as cirurgias foram realizadas por um único e experiente cirurgião (C.T.N.), com técnica cirúrgica padronizada, que consistiu em: anestesia tópica; incisão de 2,75 mm em córnea clara, auto-selante, no meridiano mais curvo; capsulorrexe circular contínua; hidrodissecção com lidocaína a $1 \%$ sem preservativos; uso de viscoelásticos dispersivo e coesivo pela técnica de "soft-shell"(24); facoemulsificação convencional, usando o sistema Infinity ${ }^{\circledR}$, e implante da LIO dentro do saco capsular, com injetor Royale ${ }^{\circledR}$ (Asico, Chicago, CA, USA) ou injetor Silver ${ }^{\circledR}$ (AMO, Santa Ana, CA, USA). Foi prescrita, no 
790 Estudo comparativo do desempenho visual e análise de frente de onda entre as lentes intra-oculares multifocais difrativas Tecnis $^{\circledR}$ ZM900 $^{\text {e AcrySof }}{ }^{\circledR}$ ReSTOR $^{\circledast}$ SN60D3

período pós-operatório, a instilação de quinolona de quarta geração (gatifloxacina $0,3 \%$ ) por 10 dias, 4 vezes ao dia, associada a corticóide tópico (dexametasona $0,1 \%$ ) a cada 2 horas ao dia inicialmente e regredido progressivamente ao longo de trinta dias.

Todos os exames de medida de acuidade visual foram realizados monocularmente, com pupilas não dilatadas, com e sem a melhor correção óptica. A acuidade visual para longe (6 m), intermediária $(70 \mathrm{~cm})$ e para perto $(33 \mathrm{~cm})$ foi medida por meio da tabela de ETDRS (Precision Vision, Aurora, Colorado, USA) com conversão em logMAR para longe e Jaeger para perto (Holladay VA conversion chart) sob condições controladas de luminosidades fotópicas estabelecidas em $85 \mathrm{~cd} / \mathrm{m}^{2}$.

A sensibilidade ao contraste foi medida com o VCTS ${ }^{\circledR} 6000$ (Vistech Consultants Incorporation, Dayton, OH, USA) sob condições fotópicas $\left(85 \mathrm{~cd} / \mathrm{m}^{2}\right)$ e mesópicas $\left(5 \mathrm{~cd} / \mathrm{m}^{2}\right)$. A tabela apresenta linhas em cinco freqüências espaciais entre 1,5 e 18 ciclos/grau (cpg). A base logarítmica 10 dos valores obtidos foram aplicadas para se obter os valores de sensibilidade ao contraste que foram usados na análise estatística.

As aberrações ópticas foram medidas pelo OPD-Scan ${ }^{\circledR}$ (Nidek Co. Ltd., Gamagori, Japan) com pupilas dilatadas farmacologicamente com tropicamida a $1 \%$, medindo pelo menos $5 \mathrm{~mm}$ de diâmetro ${ }^{(25)}$. Os diâmetros pupilares foram medidos sob as mesmas condições de iluminação pela caixa de Ginsburg (fotópica e mesópica), mediante pupilômetro infra-vermelho de Colvard (Oasis Corporation, Glendora, CA, USA).

A significância foi testada ao nível de $5 \%$ por meio dos testes de Kruskal-Wallis, Mann-Whitney e Qui-quadrado (bicaudal), ajustando-se o nível de significância, quando necessário, para $1,7 \%$.

\section{RESULTADOS}

Neste estudo, não houve nenhuma complicação intra-operatória. Não foram observadas diferenças significativas entre os grupos quanto à idade, sexo e olho operado. A média de idade foi de $65,1 \pm 2,954$ anos para o grupo Tecnis ZM900 e $62,6 \pm 3,192$ anos para o SN60D3 ( $\mathrm{p}=0,56)$. A acuidade visual média sem correção para longe foi de $+0,014 \pm 0,032 \log$ MAR $(0 \mathrm{a}+0,180)$ para o grupo Tecnis ZM900 e $+0,027 \pm 0,055$ $\log$ Mar $(0$ a 0,222) para o grupo SN60D3, sem diferença estatística ( $\mathrm{p}=0,176)$. O erro esférico foi de $-0,068 \pm 0,410 \mathrm{D}(-1 \mathrm{a}+1)$ para o grupo Tecnis ZM900 e $+0,039 \pm 0,312 \mathrm{D}(-0,25$ to $+0,5)$ para o grupo SN60D3 sem diferença estatística $(p=0,216)$.

A acuidade visual intermediária $(70 \mathrm{~cm})$ e para perto $(33 \mathrm{~cm})$ sem correção óptica foi avaliada pela tabela de ETDRS (Tabela 1), sendo verificada diferença estatística entre as lentes apenas para visão intermediária, a favor da Tecnis MF ( $\mathrm{p}<0,0001)$. Nenhum paciente necessitou de correção óptica para perto ou para longe após a cirurgia em qualquer situação do cotidiano.

A análise da sensibilidade ao contraste em condições fotópicas (Gráfico 1) revelou diferença estatisticamente significante entre as duas lentes a $6(\mathrm{p}<0,01), 12(\mathrm{p}<0,001)$ e 18 cpg $(\mathrm{p}<0,001)$ a favor da Tecnis ZM900.

\begin{tabular}{|l|cc|}
\hline $\begin{array}{c}\text { Tabela 1. Comparação da acuidade visual para perto e intermediária } \\
\text { e equivalente esférico entre a lente intra-ocular multifocal Tecnis } \\
\text { ZM900 e a ReSTOR SN60D3 }\end{array}$ \\
\multicolumn{4}{|c}{ Tecnis ZM900 } & ReSTOR SN60D3 \\
AV intermediária & $\mathrm{N}=46$ & $\mathrm{~N}=32$ \\
$\mathrm{~J} 1-\mathrm{J} 2$ & $28,26 \%(13)^{*}$ & 0 \\
$\mathrm{~J} 3-\mathrm{J} 4$ & $45,65 \%(21)^{*}$ & $6,25 \%(2)$ \\
$\mathrm{J} 5-\mathrm{J} 6$ & $17,39 \%(8)$ & $18,75 \%(6)$ \\
$>$ J6 & $8,70 \%(4)$ & $75 \%(24)^{*}$ \\
AV perto & & \\
$\mathrm{J} 1-\mathrm{J} 2$ & $95,65 \%(44)$ & $87,5 \%(28)$ \\
J3 $-\mathrm{J} 4$ & $4,34 \%(2)$ & $12,5 \%(4)$ \\
$\mathrm{J} 5-\mathrm{J} 6$ & 0 & 0 \\
$>$ J6 & 0 & 0 \\
$*$ * teste de Fisher com $\mathrm{P}<0,0001$ & & \\
\hline
\end{tabular}

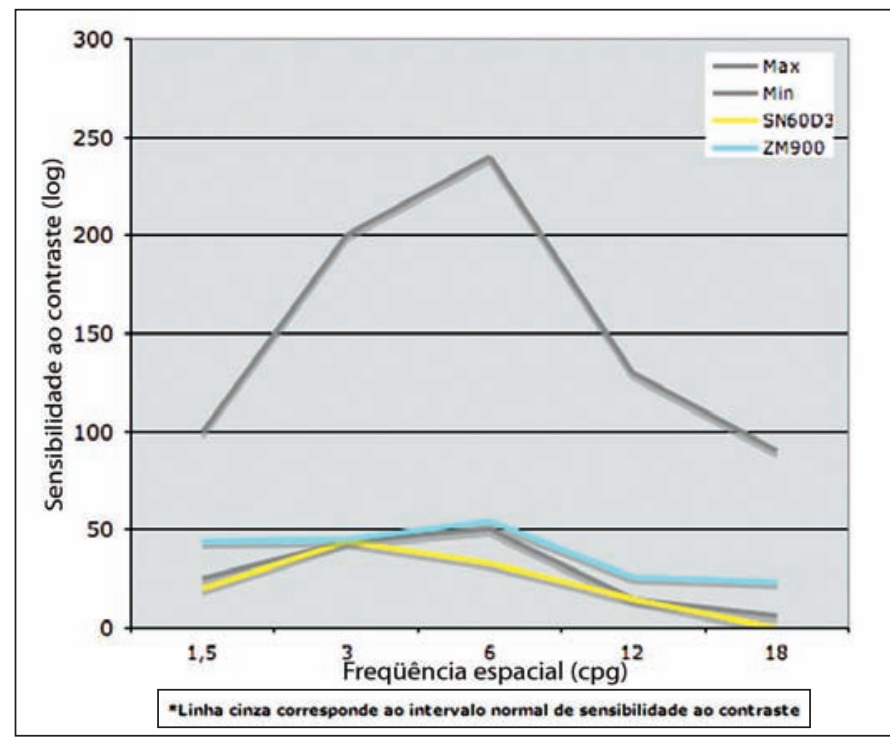

Gráfico 1 - Comparação da sensibilidade ao contraste em condições fotópicas entre as lentes intra-oculares Tecnis ZM900 ( $n=46)$ e ReSTOR SN60D3 $(n=32)$

O gráfico 2 mostra algumas variáveis que compõem as aberrações de frente de onda com OPD-Scan. As aberrações esféricas, de alta ordem e total, apresentadas pelos pacientes que receberam as lentes Tecnis ZM900 se mostrou superior à daqueles que receberam as lentes SN60D3, mas não foi observada diferença estatisticamente significante $(p>0,05)$.

\section{DISCUSSÃO}

Neste estudo, as lentes ReSTOR SN60D3 implantadas tinham pigmento amarelo com propriedades de filtro para $\mathrm{o}$ espectro azul da luz visível ${ }^{(26-27)}$. Esse tratamento poderia interferir nos exames, mas alguns autores demonstraram que não existe interferência na sensibilidade ao contraste, acuidade visual, em condições fotópicas com alta luminosidade, com ou sem ofuscamento (glare), ou mesmo na distinção das cores ${ }^{(26)}$.

A acuidade visual em alto contraste, testada por meio da tabela do ETDRS sem correção óptica para perto e para longe na 


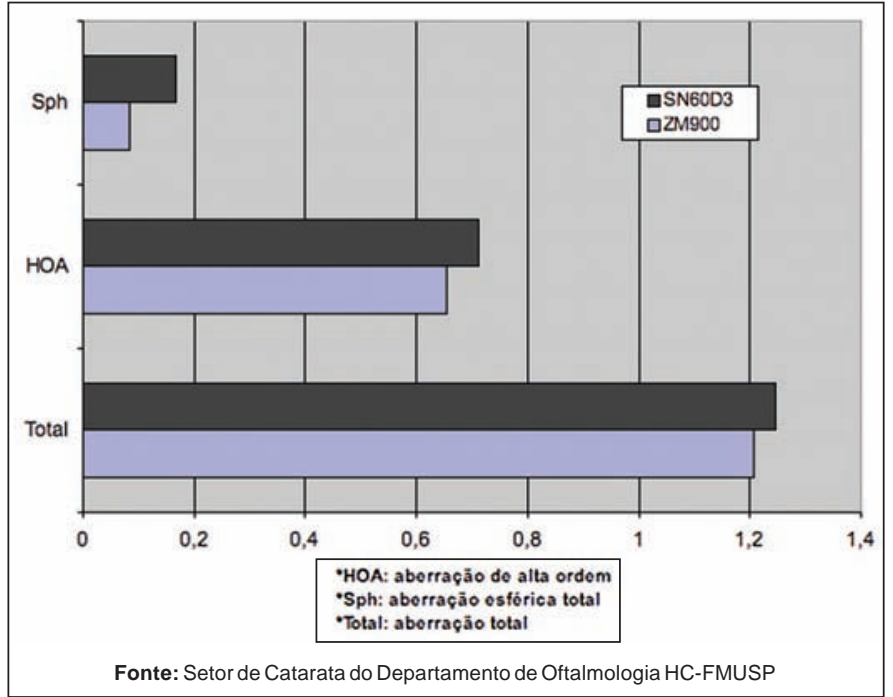

Gráfico 2 - Comparação da análise de frente de onda entre as lentes intra-oculares Tecnis MF ZM900 (n=46) e ReSTOR SN60D3 (n=32)

maioria das situações cotidianas, foi similar com ambas as LIOs neste estudo, mas houve superioridade estatisticamente significante a favor do grupo Tecnis ZM900 na visão intermediária sem correção, concordando com o critério de normalidade estabelecido e previamente descrito por diversos autores ${ }^{(14,20,28)}$.

A sensibilidade ao contraste monocular em condições fotópicas demonstrou superioridade estatisticamente significante ( $\mathrm{p}<0,0001)$ a favor do grupo Tecnis ZM900 em altas freqüências espaciais (6, 12 e $18 \mathrm{cpg})$. Alguns estudos demonstraram que a sensibilidade ao contraste em condições fotópicas foi estatisticamente diminuída com a SN60D3 ${ }^{(29)}$.

Com relação às aberrações avaliadas, houve superioridade nos valores absolutos a favor da LIO Tecnis ZM900 sobre a SN60D3, quando se analisou as aberrações totais e esféricas; entretanto, essa vantagem não foi estatisticamente significante neste estudo. Outro trabalho, com menor amostra, demonstrou redução significante das aberrações esféricas especialmente no grupo da LIO Tecnis ZM900 ${ }^{(30)}$.

Acredita-se que a superioridade do grupo da Tecnis ZM900 quanto à visão intermediária, sensibilidade ao contraste e aberrações esféricas se deva, provavelmente, à plataforma anterior asférica prolada da Tecnis ZM900 (14,20,28-30).

Em conclusão, as duas lentes intra-oculares promoveram excelente visão sem correção óptica para perto e para longe. A LIO Tecnis multifocal necessita de menos luminosidade para visão em situações de alto contraste, apresenta menos aberrações ópticas e melhor visão intermediária que a LIO ReSTOR.

\section{ABSTRACT}

Purpose: Comparison of the visual performance and wavefront analysis between patients with Tecnis ${ }^{\circledR}$ ZM900 aspheric and AcrySof ReSTOR ${ }^{\circledR}$ SN60D3 spheric diffractive multifocal intraocular lenses (IOL). Methods: This prospective comparative study included 78 eyes of 39 patients. The performed ophthalmologic evaluation included near, intermediate and distance corrected and uncorrected visual acuity, contrast sensitivity measurement (under photopic and mesopic conditions) and wavefront analysis with the OPD-Scan aberrometrer. Results: Uncorrected near and distance visual acuity and aberrometry analysis were similar with both intraocular lenses; nevertheless, intermediate visual acuity and contrast sensitivity under photopic conditions were statistically better with Tecnis ZM900. Conclusion: Both studied intraocular lenses promoted excellent near and distance postoperative visual acuity, in high contrast situations. Tecnis ZM900 IOL provided good quality of vision with high contrast condition in low luminosity with reduction in spherical aberration and improvements in intermediate visual acuity when compared to ReSTOR IOL.

Keywords: Lenses, intraocular; Contrast sensitivity; Vision; Visual acuity

\section{REFERÊNCIAS}

1. Pepose JS, Qazi MA, Davies J, Doane JF, Loden JC, Sivalingham V, Mahmoud AM. Visual performance of patients with bilateral vs combination Crystalens, ReZoom, and ReSTOR intraocular lens implants. Am J Ophthalmol. 2007; 144(3):347-57. Comment in: Am J Ophthalmol. 2008;145(4):593-4.

2. Mester U, Hunold W, Wesendahl T, Kaymak H. Functional outcomes after implantation of Tecnis ZM900 and Array SA40 multifocal intraocular lenses. J Cataract Refract Surg. 2007;33(6):1033-40.

3. Maliugin BE, Fedorova IS, Antonian SA, Sobolev NP, Tsyplakova TS. [Surgical correction of presbyopia with multifocal diffractive intraocular lenses]. Vestn Oftalmol. 2007;123(4):3-6. Russian

4. López-Gil N, Montés-Micó R. New intraocular lens for achromatizing the human eye. J Cataract Refract Surg. 2007;33(7):1296-302. Erratum in: J Cataract Refract Surg. 2008;34(7):1058.

5. Jacobi FK, Kessler W, Held S. [Optical performance of multifocal intraocular lenses. Investigation of the Array SA40N vs. Acri.Twin at the "physical eye" according to Reiner and Jacobi]. Ophthalmologe. 2007;104(3):236-42. German.

6. Fernández-Vega L, Alfonso JF, Rodríguez PP, Montés-Micó R. Clear lens extraction with multifocal apodized diffractive intraocular lens implantation. Ophthalmology. 2007;114(8):1491-8.

7. Obuchowska I, Mariak Z. [Sir Harold Ridley-the creator of modern cataract surgery]. Klin Oczna. 2005;107(4-6):382-4. Polish.

8. Alió JL, Schimchak P, Negri HP, Montés-Micó R. Crystalline lens optical dysfunction through aging. Ophthalmology. 2005;112(11):2022-9. Comment in: Ophthalmology. 2007;114(3):618; author reply 619.

9. Rawer R, Stork W, Spraul CW, Lingenfelder C. Imaging quality of intraocular lenses. J Cataract Refract Surg. 2005;31(8):1618-31.

10. Nio YK, Jansonius NM, Geraghty E, Norrby S, Kooijman AC. Effect of intraocular lens implantation on visual acuity, contrast sensitivity, and depth of focus. J Cataract Refract Surg. 2003;29(11):2073-81.

11. Marcos S, Barbero S, Jiménez-Alfaro I. Optical quality and depth-of-field of eyes implanted with spherical and aspheric intraocular lenses. J Refract Surg. 2005;21(3):223-35.

12. Friström B, Lundh BL. Colour contrast sensitivity with different intraocular lens materials in the right and left eyes in same day surgery. Acta Ophthalmol Scand. 2005;83(4):443-7.

13. Rodríguez-Galietero A, Montés-Micó R, Muñoz G, Albarrán-Diego C. Comparison of contrast sensitivity and color discrimination after clear and yellow intraocular lens implantation. J Cataract Refract Surg. 2005;31(9):1736-40.

14. Oliveira F, Muccioli C, Silva LMP, Soriano ES, Souza CEB, Belfort Júnior R. Avaliação da sensibilidade ao contraste e da estereopsia em pacientes com lente intraocular multifocal. Arq Bras Oftalmol. 2005;68(4):439-43.

15. Bellucci R, Scialdone A, Buratto L, Morselli S, Chierego C, Criscuoli A, et al. Visual acuity and contrast sensitivity comparison between Tecnis and AcrySof SA60AT intraocular lenses: A multicenter randomized study. J Cataract Refract Surg. 2005;31(4):712-7. Erratum in: J Cataract Refract Surg. 2005;31(10):1857. 
792 Estudo comparativo do desempenho visual e análise de frente de onda entre as lentes intra-oculares multifocais difrativas Tecnis $^{\circledR}$ ZM900 $^{\text {e AcrySof }}{ }^{\circledR}$ ReSTOR $^{\circledast}$ SN60D3

16. Dietze HH, Cox MJ. Limitations of correcting spherical aberration with aspheric intraocular lenses. J Refract Surg. 2005;21(5):S541-6.

17. Guirao A, Redondo M, Geraghty E, Piers P, Norrby S, Artal P. Corneal optical aberrations and retinal image quality in patients in whom monofocal intraocular lenses were implanted. Arch Ophthalmol. 2002;120(9):1143-51.

18. Davison JA, Simpson MJ. History and development of the apodized diffractive intraocular lens. J Cataract Refract Surg. 2006;32(5):849-58.

19. Sallet G. Refractive outcome after bilateral implantation of an apodized diffractive intraocular lens. Bull Soc Belge Ophtalmol. 2006;(299):67-73.

20. Kohnen T, Allen D, Boureau C, Dublineau P, Hartmann C, Mehdorn E, et al. European multicenter study of the AcrySof ReSTOR apodized diffractive intraocular lens. Ophthalmology. 2006;113(4):584.e1.

21. Werner W, Roth EH. [Image properties of spherical as aspheric intraocular lenses]. Klin Monatsbl Augenheilkd. 1999;214(4):246-50. German.

22. Rekas M, Zelichowska B. Multifocal diffractive intraocular lenses in cataract surgery-preliminary report. Klin Oczna. 2006;108(4-6):186-90.

23. Olsen T, Nielsen PJ. Immersion versus contact technique in the measurement of axial length by ultrasound. Acta Ophthalmol (Copenh). 1989;67(1):101-2.

24. Auffarth GU, Holzer MP, Visessook N, Apple DJ, Völcker HE. Removal times for a dispersive and a cohesive ophthalmic viscosurgical device correlated with intraocular lens material. J Cataract Refract Surg. 2004;30(11):2410-4.
25. Jankov MR $2^{\text {nd }}$, Iseli HP, Bueeler M, Schor P, Seiler T, Mrochen M. The effect of phenylephrine and cyclopentolate on objective wavefront measurements. J Refract Surg. 2006;22(5):472-81.

26. Marshall J, Cionni RJ, Davison J, Ernest P, Lehmann R, Maxwell WA, Solomon K. Clinical results of the blue-light filtering AcrySof Natural foldable acrylic intraocular lens. J Cataract Refract Surg. 2005;31(12):2319-23. Comment in: J Cataract Refract Surg. 2005;31(12):2237-8.

27. Mayer S, Wirbelauer C, Pham DT. [Functional results after intraocular lens implantation with or without blue light filter: an intraindividual comparison] Klin Monatsbl Augenheilkd. 2006;223(2):142-6. German.

28. Souza CE, Gerente VM, Chalita MR, Soriano ES, Freitas LL, Belfort R Jr. Visual acuity, contrast sensitivity, reading speed, and wavefront analysis: pseudophakic eye with multifocal IOL (ReSTOR) versus fellow phakic eye in non-presbyopic patients. J Refract Surg. 2006;22(3):303-5.

29. Souza CE, Muccioli C, Soriano ES, Chalita MR, Oliveira F, Freitas LL, et al. Visual performance of AcrySof ReSTOR apodized diffractive IOL: a prospective comparative trial. Am J Ophthalmol. 2006;141(5):827-32.

30. Toto L, Falconio G, Vecchiarino L, Scorcia V, Di Nicola M, Ballone E, Mastropasqua L. Visual performance and biocompatibility of 2 multifocal diffractive IOLs: six-month comparative study. J Cataract Refract Surg. 2007;33(8):1419-25. Comment in: J Cataract Refract Surg. 2008;34(4):528; author reply 528-9. 